\title{
Measuring Reaction Rate Constant in Individual Cells to Facilitate Accurate Analysis of Cell-Population Heterogeneity
}

\author{
Vasilij Koshkin, Sven Kochmann, Apinaya Sorupanathan, Chun Peng, Laurie E. Ailles, Geoffrey Liu, \\ and Sergey N. Krylov*
}

\begin{abstract}
We propose Cytometry of Reaction Rate Constant (CRRC) for accurate analysis of cell-population heterogeneity with respect to a specific molecular reaction. Conceptually, in CRRC, the cells are loaded with a reaction substrate, and its conversion into a product is followed by time-lapse fluorescence microscopy at the single-cell level. A reaction rate constant is determined for every cell by using a known kinetic mechanism of the reaction, and a kinetic histogram "number of cells vs. the rate constant" is built. Finally, this histogram is used to determine parameters of reaction-based cell-population heterogeneity. Here, we studied a reaction of substrate extrusion from cells by $A B C$ transporters. We proved that sizes of subpopulations with different extrusion rates could be accurately determined from the kinetic histogram, and this determination was not significantly affected by change in substrate concentration. We foresee that CRRC will facilitate the development of reliable disease biomarkers based on parameters of reaction-based cell-population heterogeneity.
\end{abstract}

Cell populations within the same tissue are inherently heterogeneous, and this heterogeneity may be so extensive that distinct subpopulations become identifiable. ${ }^{[1]}$ For example, populations of cancer cells are comprised typically of at least two subpopulations: bulk tumor cells (that are often sensitive to chemotherapy) and tumor-initiating cells (that are often chemotherapy-resistant). ${ }^{[2]} \quad$ In $\quad$ general, cell-population heterogeneity is caused by differences in molecular reactions between the cells. When a specific reaction is associated with cellpopulation heterogeneity, it can serve as a basis for characterizing this heterogeneity. ${ }^{[3]}$ Cell-population heterogeneity with respect to a specific molecular reaction has been proposed and used for development of disease biomarkers. ${ }^{[4]}$ However, such biomarkers proved to be unreliable clinically due to their inadequate accuracy. ${ }^{[5]}$ Inaccuracy of these biomarkers can be caused either by a weak link between the disease and the reaction-based cellpopulation heterogeneity or by an inaccurate method used to characterize this heterogeneity, or due to both reasons.

This work was motivated by our insight that the two approaches currently used for characterizing reaction-based cell-

[*] Dr. V. Koshkin, Dr. S. Kochmann, A. Sorupanathan,

Prof. C. Peng, Prof. S. N. Krylov

Centre for Research on Biomolecular Interactions, York University,

Toronto, Ontario M3J 1P3, Canada

E-mail: skrylov@yorku.ca

Homepage: http://www.yorku.ca/skrylov/

Prof. L. E. Ailles

Department of Medical Biophysics, University of Toronto, Toronto, Ontario, Canada N5G 1 L7

Prof. G. Liu

Department of Medicine, Medical Oncology, Princess Margaret

Cancer Centre, Toronto, Ontario, Canada M5G 2M9 population heterogeneity are inherently inaccurate due to the nonkinetic nature of measures used to characterize the reaction. The first approach is based on classical flow cytometry which is limited to interrogating every cell only once. ${ }^{[6]}$ Accordingly a measure of reaction extent is a fluorescence intensity from the intracellular substrate, and parameters of cell-population heterogeneity are obtained from a non-kinetic histogram: "number of cells vs fluorescence intensity". ${ }^{[7]}$ The second approach is based on fluorescence microscopy and involves measurements of fluorescence signal intensity for each cell more than once. ${ }^{[8]}$ The results are, however, typically analyzed by using only two intensities: $I_{1}$ and $I_{2}$ measured at two different times. ${ }^{[9]}$ Parameters of cell-population heterogeneity in such analysis are obtained from a non-kinetic histogram, e.g.: "number of cells $v s\left|I_{2}-I_{1}\right| / I_{1}$ " ${ }^{[10}$ Thus, both current approaches utilize non-kinetic measures of reaction extent and determine quantitative characteristics of reaction-based cell-population heterogeneity from non-kinetic histograms.

We hypothesize that the non-kinetic histograms can provide largely inaccurate parameters of reaction-based cell-population heterogeneity. This hypothesis translates to a notion that nonkinetic approaches should not be used for the development of disease biomarkers that are built upon reaction-based cellpopulation heterogeneity. In contradistinction, we further postulate that accurate analysis of such heterogeneity requires a kinetic approach built upon a kinetic measure: a reaction rate constant. Importantly, measuring a reaction rate constant at the single-cell level is feasible provided that there is a fluorescent or fluorogenic reaction substrate and the kinetic mechanism of the reaction is known. ${ }^{[11]}$

We coin the term, Cytometry of Reaction Rate Constant (CRRC), to describe the kinetic characterization of reaction-based cell-population heterogeneity. The concept of CRRC is schematically depicted in Figure 1. First, the cells are loaded with a fluorescent (or fluorogenic) substrate, and the substrate is naturally involved into the cellular reaction of interest. Second, kinetics of change of intracellular substrate concentration is followed by imaging a large number of cells microscopically and measuring the fluorescence intensity in individual cells as a function of time. Third, the reaction rate constant is determined for every cell from kinetic data by using a known kinetic mechanism of the reaction (note that CRRC is only applicable to reactions with confirmed kinetic mechanisms). Fourth, the rate constant values are used to construct a kinetic histogram: "number of cells vs. the rate constant". Finally, the kinetic histogram is used to determine parameters of reaction-based cell-population heterogeneity, such as the number and sizes of distinct subpopulations comprising the heterogeneous cell population. The goal of this work is to examine experimentally the ability of CRRC to determine accurate parameters of reaction-based cell-population heterogeneity.

The reaction that we have chosen to study is substrate extrusion from cells by membrane proteins known as ATP-binding cassette transporters (ABC transporters). ${ }^{[12]}$ This reaction is a driving force of multi-drug resistance (MDR) of cancer cells and is usually 


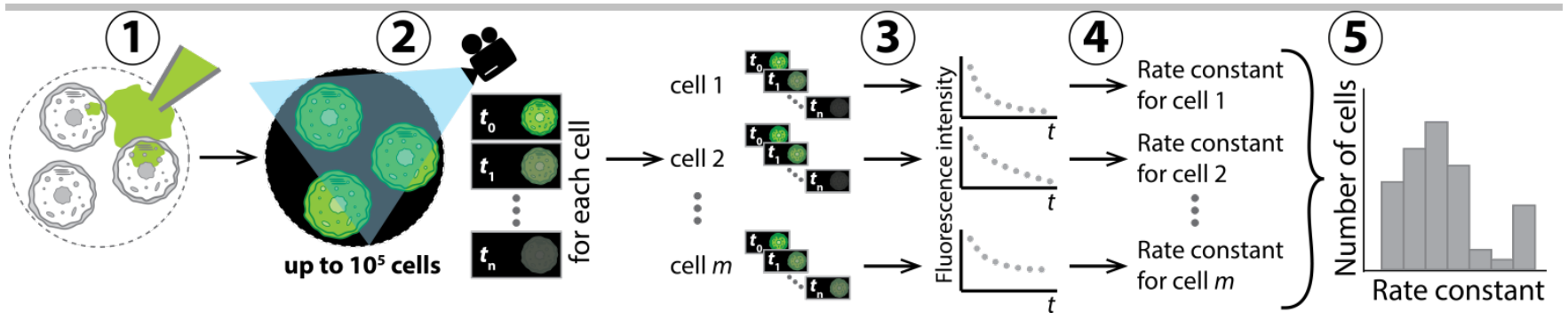

Figure 1. Schematic depiction of Cytometry of Reaction Rate Constant. See text for details.

called MDR transport. ${ }^{[13]}$ ABC transporters have low substrate specificity and, therefore, fluorophores can be used as substrates in studies of MDR transport. ${ }^{[14]}$ Non-kinetic histograms were used to characterize MDR-based heterogeneity of cancer cell populations; the size of a cell subpopulation with increased MDR transport activity was used as a heterogeneity parameter to develop predictive biomarkers of clinical resistance of cancer to chemotherapy. ${ }^{[15]}$ All such biomarkers, however, have proven to be unreliable clinically, ${ }^{[16]}$ likely due to the inaccuracy of the nonkinetic analysis employed for their derivation. Hence, finding an accurate approach for characterizing MDR-based cell-population heterogeneity may have significant clinical benefits. The medical importance of the MDR process served as a major justification for our use of this reaction in this methodological study.

Mechanistically, an ABC transporter (T) binds the substrate (S) on the inner side of the membrane, "turns" around, and releases $S$ on the outer side of the membrane (Figure S1). ${ }^{[17]}$ The MDR transport proceeds through the formation of an intermediate complex (TS) and, thus, can be described by the Michaelis-Menten equation (commonly used for enzymatic reactions): ${ }^{[18]}$

$$
\mathrm{T}+\mathrm{S}_{\mathrm{in}} \underset{k_{-1}}{\stackrel{k_{1}}{\rightleftarrows}} \mathrm{TS} \stackrel{k_{2}}{\longrightarrow} \mathrm{T}+\mathrm{S}_{\text {ex }}
$$

where $S_{\text {in }}$ is intracellular substrate and $S_{\text {ex }}$ is extracellular substrate. This process can be characterized by a unimolecular rate constant of MDR transport, $k_{\mathrm{MDR}}$, which is a ratio between the maximum reaction rate, $V_{\max }$, and the Michaelis constant, $K_{\mathrm{M}}$ : $k_{\mathrm{MDR}}=V_{\max } / K_{\mathrm{M}} \cdot{ }^{[19]}$ Our earlier work has proven that the single-cell kinetics of MDR follow the Michaelis-Menten mechanism, ${ }^{[20]}$ and, thus, $k_{\mathrm{MDR}}$ (as defined above) is suitable for the analysis of MDRbased cell-population heterogeneity by CRRC.

The timeline of our experiment is schematically shown in Figure 2 (for a single cell). The experiment starts with cells being in a Petrie dish on a microscope stage. A fluorescent substrate of

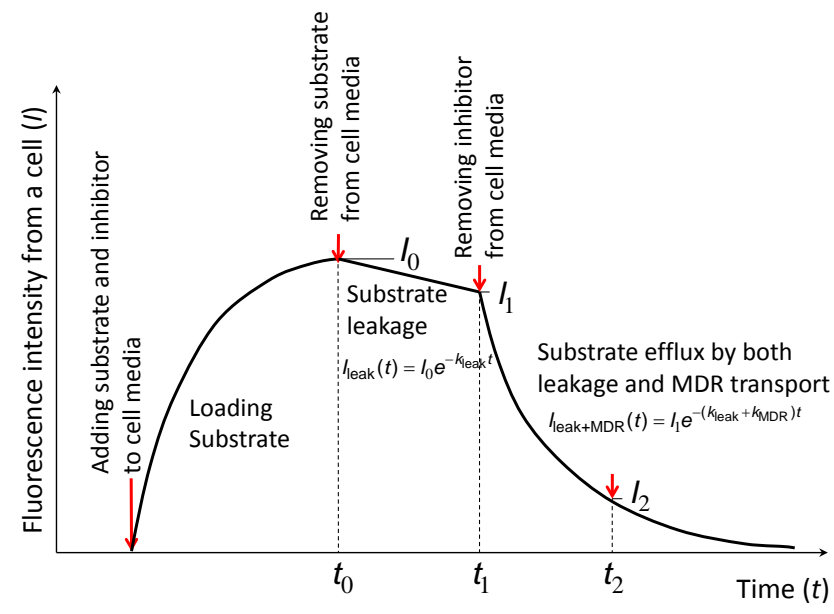

Figure 2. Schematic depiction of the timeline of CRRC experiment aiming to determine the rate constant of MDR efflux, $k_{\text {MDR }}$. See text for details.
MDR transport and an inhibitor of ABC transporters are added simultaneously to the cell media to initiate substrate loading into the cells. When fluorescence intensity from a cell approaches its saturation at level $I_{0}$, the substrate loading is stopped by removing the extracellular substrate while keeping the inhibitor in the media. This initiates passive substrate leakage through the membrane, accompanied by fluorescence intensity decrease to level $I_{1}$. Then the MDR-mediated efflux is initiated by removing the MDR inhibitor, and the cumulative substrate efflux by both its leakage and MDR transport is followed.

A kinetic curve of substrate efflux consists of an initial segment (from $t_{0}$ to $t_{1}$ ) representing passive substrate leakage from the cell and the main segment (after $t_{1}$ ) that corresponds to the cumulative effect of the leakage and MDR transport. Substrate leakage from a cell is accompanied by exponential decrease of cellular fluorescence intensity:

$$
I_{\text {leak }}(t)=I_{0} e^{-k_{\text {leak }} t}
$$

where $I_{0}$ is the initial intensity and $k_{\text {leak }}$ is a unimolecular rate constant characterizing the leakage process. Fluorescence intensity decreases to $I_{1}$ relatively slowly so that $\left(I_{0}-I_{1}\right) / I_{0}<<1$. This allows the calculation of $k_{\text {leak }}$ from the near linear initial segment using the following approximate equation:

$$
k_{\text {leak }} \approx \frac{I_{0}-I_{1}}{I_{0}} \frac{1}{\left(t_{1}-t_{0}\right)}
$$

This value of $k_{\text {leak }}$ can then be used to determine $k_{\mathrm{MDR}}$ from the main exponential segment of the kinetic trace. The cumulative efflux is accompanied by exponential decrease of cellular fluorescence intensity with a cumulative rate constant $k_{\text {leak }}+k_{\mathrm{MDR}}$ :

$$
I_{\text {leak }+\mathrm{MDR}}(t)=I_{1} e^{-\left(k_{\text {leak }}+k_{\mathrm{MDR}}\right) t}
$$

Note that $I_{0}, k_{\text {leak }}, I_{1}$, and $k_{\mathrm{MDR}}$ are unique for every cell. The value of $k_{\mathrm{MDR}}$ for a single cell is determined from a single kinetic curve by finding $k_{\text {leak }}$ from eq. (3) and placing it in eq. (4), which is then used to fit the main segment on this kinetic curve with $k_{\mathrm{MDR}}$ being a varying parameter. If $k_{\text {leak }}<<k_{\mathrm{MDR}}$, then the procedure of $k_{\mathrm{MDR}}$ determination can be simplified by neglecting $k_{\text {leak }}$ and finding $k_{\mathrm{MDR}}$ from the main segment on the kinetic trace (after $t_{1}$ ) using the following simple equation:

$$
I_{\mathrm{MDR}}(t)=I_{1} e^{-k_{\mathrm{MDR}} t}
$$

Accuracy of the kinetic analysis was studied in comparison to that of the non-kinetic analysis of the same data set. A kinetic curve utilized for finding $k_{\mathrm{MDR}}$ (see Figure 2) was also used to calculate a non-kinetic measure of MDR transport, $\left|I_{2}-I_{1}\right| / I_{1}$, and construct the non-kinetic histogram "number of cells $v s\left|I_{2}-I_{1}\right| / I_{1}$ ". The value of $\left|I_{2}-I_{1}\right| / I_{1}$ corresponds to a fraction of the MDR substrate extruded from the cell.

In this study, we used two types of A2780 cultured ovarian cancer cells: a drug-sensitive parental cell line with a basal MDR activity and its derivative drug-resistant subline with increased MDR activity. ${ }^{[21]}$ The drug-sensitive line models bulk tumor cells, while the drug-resistant subline models tumor-initiating cells. ${ }^{[22]}$ 


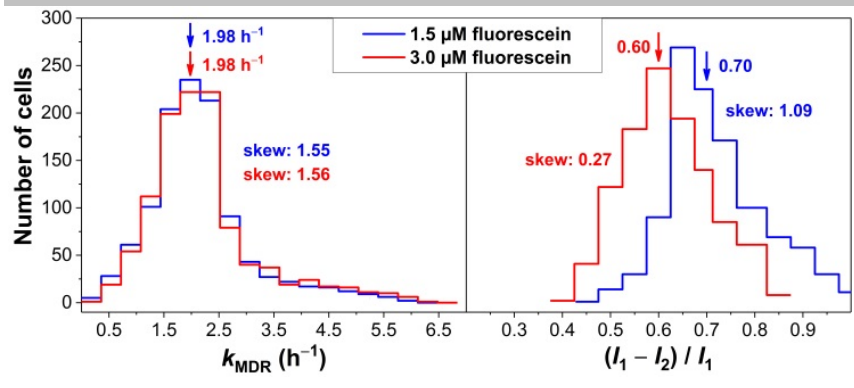

Figure 3. The effect of substrate concentration on kinetic (left) and non-kinetic (right) histograms obtained from single-cell time dependence of intracellular fluorescence of MDR substrate. The arrows indicate positions of median $k_{\text {MDR }}$ and $\left(I_{1}-I_{2}\right) / I_{1}$ for the respective peaks.

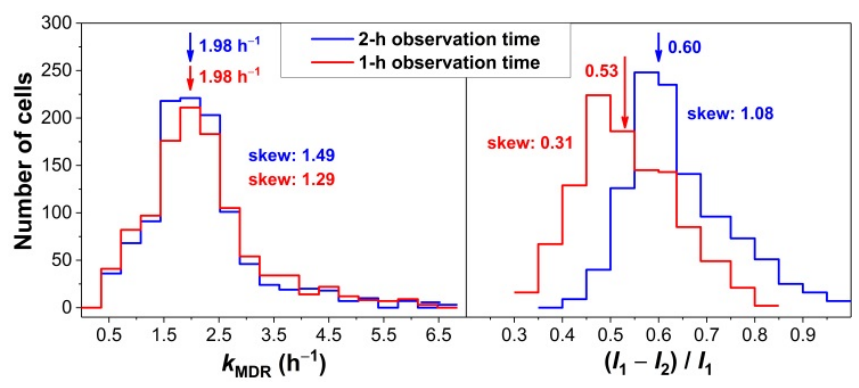

Figure 4. The effect of observation time on kinetic (left) and non-kinetic (right) histograms obtained from single-cell time dependence of intracellular fluorescence of MDR substrate. The arrows indicate positions of median $k_{\mathrm{MDR}}$ and $\left(I_{1}-I_{2}\right) / I_{1}$ for the respective peaks.

Fluorescein was used as a substrate of MDR transport (a known substrate for the MRP-type ABC transporters predominantly expressed in these cells), ${ }^{[23]}$ and glyburide was used as an inhibitor of MRP transporters. ${ }^{[2]}$ The first-order regime of substrate efflux, which is required for applicability of eq. (4), was ensured by using concentrations of fluorescein $(1.5$ and $3.0 \mu \mathrm{M})$ much lower than the value of $K_{\mathrm{M}}(14-247 \mu \mathrm{M}){ }^{[23,25]}$

Time-lapse fluorescence imaging of all cells in the field of view with 3-min intervals started after replacing fluoresceincontaining media with fluorescein-free media $\left(t_{0}\right.$ in Figure 2$)$ and ended after 2-3 h. Imaging was performed with an Olympus Fluoview FV300 laser scanning confocal fluorescence microscope, using an argon-ion laser $\left(\lambda_{\mathrm{exc}}=488 \mathrm{~nm}\right.$; excitation power: $\left.10 \mathrm{~mW}\right)$ and an Omega Optical XF75 filter set. Images were acquired with an open pinhole to collect signal from the whole depth of the cell. Details on image-acquisition procedures can be found in the Supporting Information.

Individual cells were identified in every image and traced through the entire time series. Mean fluorescence intensity for every cell in a single image was calculated and used to create a kinetic curve for every cell (see Supporting Information for details of image processing). We found that for our experimental system $k_{\text {leak }}<0.1 \times k_{\mathrm{MDR}}$, and therefore, eq. (5) was used for finding $k_{\mathrm{MDR}}$. The values of $k_{\mathrm{MDR}}$ and $\left(I_{1}-I_{2}\right) / I_{1}$ were found as described above and used to construct kinetic and non-kinetic histograms, respectively. The above-described procedures were used for three sets of experiments that assessed the quantitative performance of the kinetic analysis in comparison to that of the non-kinetic one.

In the first set of experiments, we compared robustness of the kinetic and non-kinetic analyses in characterizing heterogeneity of a unimodal histogram (containing only a single peak) obtained for the drug-sensitive cell line. Substrate concentration and observation time are typical variable parameters; ${ }^{[26]}$ therefore, we studied the sensitivity of analysis results to changes in fluorescein concentration and observation time. The parental drug-sensitive cell line was used in this part of our study.

To understand analysis sensitivity to substrate concentration, we utilized two fluorescein concentrations in the cell media, 1.5 and $3.0 \mu \mathrm{M}$, which provided sufficient intensity of fluorescence and were below $K_{\mathrm{M}}(14-247 \mu \mathrm{M}) \cdot{ }^{[25]}$ Observation time of $2 \mathrm{~h}$ is common for MDR assays; ; $^{[2]}$ it was used as a default value throughout this study. For illustrative purposes, examples of images and kinetic traces are shown in Supporting Information. Fluorescence images of $>1000$ cells each were processed (kinetic traces for all cells are archived in the Supporting Information) and kinetic and non-kinetic histograms were plotted (Figure 3). As expected for a pure cell line, both histograms were unimodal and could be characterized by the median values of $k_{\mathrm{MDR}}$ and $\left(I_{1}-I_{2}\right) / I_{1}$, respectively, and peak skewness, which is a measure of peak asymmetry. ${ }^{[28]}$ Doubling the substrate concentration did not cause significant changes in the kinetic histogram (Figure 3, left): the median value of $k_{\mathrm{MDR}}$ as well as peak skewness did not change significantly ( $k_{\mathrm{MDR}}$ : both $1.98 \mathrm{~h}^{-1}$; skewness: 1.55 and 1.56). In contrast, the non-kinetic analysis (Figure 3, right) revealed a great dependence of peak parameters on the substrate concentration. Upon increasing fluorescein concentration from 1.5 to $3.0 \mu \mathrm{M}$, the median value of $\left(I_{1}-I_{2}\right) / I_{1}$ decreased over $10 \%$ (from 0.70 to 0.60 ) while skewness dropped by a factor of 4 (from 1.09 to 0.27 ). Thus, the kinetic analysis was much more robust to changing substrate concentration than its non-kinetic version.

We then compared robustness of the kinetic and non-kinetic analyses with regards to shortening the observation time in the assay. In general, shortening the observation time is beneficial as it allows for a faster assay. Accordingly, the values of $k_{\mathrm{MDR}}$ and $\left(I_{1}-I_{2}\right) / I_{1}$ were determined from the default 2-h kinetic traces and from truncated 1-h traces for each of $>1000$ cells (archived in the Supporting Information), and kinetic, and non-kinetic histograms were constructed for 2-h and 1-h observation times (Figure 4). The results were similar to those for the different substrate concentrations. The effects of changing observation time on the median value of the MDR transport measure and on peak skewness were much greater for the non-kinetic analysis (Figure 4, right) than for the kinetic analysis (Figure 4, left). To summarize, the results of experiments with different substrate concentrations and different observation times proved that the kinetic CRRC analysis, in contrast to its non-kinetic counterpart, was robust to variations in these assay conditions, when used to determine parameters of a unimodal distribution in the histogram.

In the second set of experiments, we assessed the ability of the kinetic and non-kinetic analyses to distinguish cells with different levels of MDR activity, namely to resolve the drug-sensitive cell line from the drug-resistant subline. The concentration of substrate was $1.5 \mu \mathrm{M}$ and the observation time was $2 \mathrm{~h}$. Single-cell kinetic traces were recorded for the drug-sensitive line and drug-resistant subline ( $>1000$ cells each), in two separate experiments (archived in the Supporting Information). The values of $k_{\mathrm{MDR}}$ and $\left(I_{1}-I_{2}\right) / I_{1}$ were found as described above and used to construct kinetic and non-kinetic histograms, respectively, for both types of cells (Figure 5). The median values of $k_{\mathrm{MDR}}$ for the resistant and sensitive cells differed by a factor of 2.3. The median value of $\left(I_{1}-I_{2}\right) / I_{1}$ for resistant and sensitive cells differed only by a factor of 1.2. Resolution $R$ of peaks corresponding to drug-resistant and drugsensitive cells was defined as:

$$
R=\frac{\left|M_{1}-M_{2}\right|}{\frac{1}{2}\left(W_{1}+W_{2}\right)}
$$




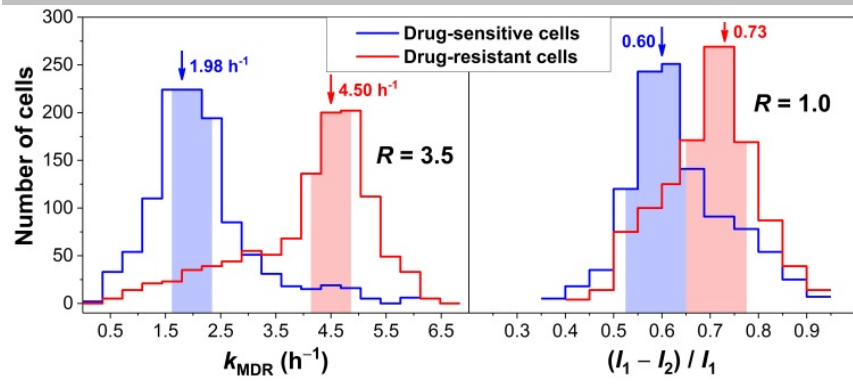

Figure 5. Resolution of peaks corresponding to the drug-sensitive cell line (with basal MDR activity) and drug-resistant subline (with elevated MDR activity) in kinetic (left) and non-kinetic (right) histograms obtained from singlecell time dependence of intracellular fluorescence of MDR substrate. The vertical arrows indicate positions of median values of $k_{\mathrm{MDR}}$ and $\left(I_{1}-I_{2}\right) / I_{1}$ and the shaded areas show interquartile ranges for the respective peaks.

where $M_{1}$ and $M_{2}$ are median values of the two distributions (drugsensitive and drug-resistant) and $W_{1}$ and $W_{2}$ are their widths calculated as interquartile ranges. ${ }^{[28]}$ Based on the histograms in Figure 5, resolution between the peaks in the kinetic assay was 3.5; in the non-kinetic assay it was only 1.0. These results proved that the kinetic assay had a greater resolving power for cell populations with different reaction rates.

In the third and final set of experiments, we assessed the ability of kinetic and non-kinetic analyses to determine accurately the size of a small subpopulation of cells with a reaction rate distinct from that of a larger subpopulation. A bimodal cell population required for such a study was prepared by mixing $80 \%$ of the drug-sensitive cells with $20 \%$ of the drug-resistant cells. The experiments for the bimodal cell populations were conducted for two different substrate concentrations, 1.5 and $3.0 \mu \mathrm{M}$, to test analysis robustness with regards to the varying substrate concentration.

Fluorescence images were processed to determine $k_{\mathrm{MDR}}$ and $\left(I_{1}-I_{2}\right) / I_{1}$ for each of 791 cells (archived in the Supporting Information) and the corresponding kinetic and non-kinetic histograms were plotted (Figure 6). Since the drug-resistant subline had 2.3 times higher $k_{\mathrm{MDR}}$, the kinetic histogram was bimodal for both concentrations of fluorescein. The relative area of the peak with higher $k_{\mathrm{MDR}}$ is defined by the fraction of drug-resistant cells in the cell population, which was $20 \%$ in our experiments. The measured values of this relative area were $19 \%$ and $18 \%$ for 1.5 and $3.0 \mu \mathrm{M}$ fluorescein, respectively. A deviation of less than $10 \%$ from the expected value proved that the kinetic analysis was both accurate and robust with regards to variations in the substrate concentration. In contrast, the non-kinetic analysis of the size of drug-resistant subpopulation revealed both inaccuracy and nonrobustness. The measured size was $13 \%$ for $1.5 \mu \mathrm{M}$ fluorescein (over $30 \%$ difference from the actual size), while the size could not be determined at all for $3.0 \mu \mathrm{M}$ fluorescein due to the lack of a clear boundary between the drug-sensitive and drug-resistant peaks in this non-kinetic histogram (the distribution was unimodal for 3.0 $\mu \mathrm{M}$ fluorescein). Thus, the kinetic analysis of the size of a subpopulation of cells with a distinct MDR transport rate was accurate and robust while the non-kinetic analysis lacked both accuracy and robustness.

In conclusion, we proved that CRRC could accurately determine parameters of cell-population heterogeneity for unimodal and bimodal distributions in the "number of cells vs reaction rate constant” histogram. In addition, the results were not affected significantly by variations in substrate concentration or observation time. The accuracy and robustness of CRRC suggest it should be the preferred method for discovery and validation of disease biomarkers based on cell-population heterogeneity.
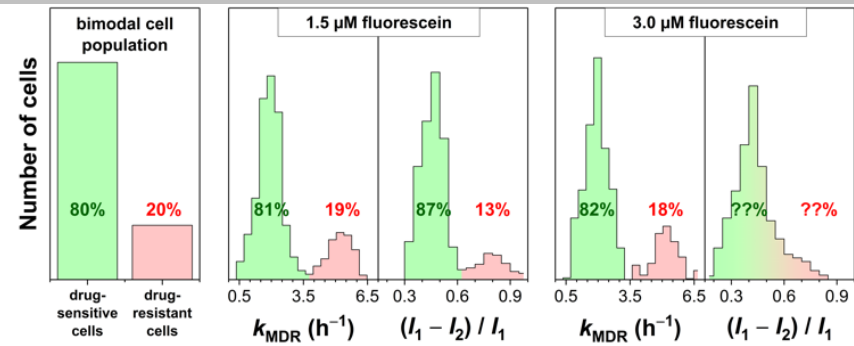

Figure 6. Quantitation of the relative size of drug-resistant cells in a bimodal cell population consisting of both drug-resistant and drug-sensitive cells. The left panel illustrates the composition of the bimodal cell population which was made by mixing drug-resistant and drug-sensitive cells at a 1:4 ratio. The middle and right panels shows the comparison of kinetic and non-kinetic histograms for 1.5 and 3.0 $\mu \mathrm{M}$ fluorescein. Question marks reflect an inability to distinguish between cell populations.

We coin the term, MDRmetry, for the adaptation of CRRC to assess the MDR-based heterogeneity that is outlined in this study. MDRmetry will be important practically for development of accurate predictors of MDR-mediated chemoresistance in cancer. Similar adaptations can be made potentially for the other two major cellular defence mechanisms contributing to chemoresistance, namely, drug degradation by intracellular enzymatic cascades and repair of drug-induced DNA damage by nuclear enzymes. ${ }^{[29]}$

It is important to emphasize that CRRC requires that a kinetic mechanism of the reaction of interest be known; in essence, this necessitates a separate experimental study to confirm this mechanism. Some research on kinetic mechanisms of clinicallyrelevant cellular reactions has already been conducted. ${ }^{[30]}$ We expect that our results will convince equipped laboratories to focus on such kinetic studies. Modern microscopes and image processing software allow automation of CRRC for fast and reliable acquisition of kinetic data for thousands of cells, a requirement for determining statistical significance of the heterogeneity parameters. The availability of required hardware and software suggests that the use of CRRC can gain momentum rapidly.

\section{Conflict of interest}

The authors declare no conflict of interest.

\section{Acknowledgements}

This work was supported by an NSERC Discovery grant No. 238990.

Keywords: $\underline{\text { Bioinformatics }} \cdot \underline{\text { Biological activity }} \cdot \underline{\text { Kinetics }} \bullet$ Cell heterogeneity $\bullet$ Cytometry of Reaction Rate Constant

[1] a) S. Huang, Development 2009, 136, 3853; b) S. J. Altschuler, L.F. Wu, Cell 2010, 141, 559.

[2] a) M. J. Kwon, Y. K. Shin, Int. J. Mol. Sci. 2013, 14, 6624; b) K. McIntosh, C. Balch, A.K. Tiwari, Expert Opin. Drug Metab. Toxicol. 2016, 12, 633.

[3] N. V. Mantzaris, Biophys. J. 2007, 92, 4271.

[4] a) F. Niu, D. C. Wang, J. Lu, W. Wu, X. Wang, J. Cell. Mol. Med. 2016, 20, 1789; b) E. Wambre, V. Bajzik, J. H. DeLong, K. O’Brien, Q.-Anh Nguyen, C. Speake, V. H. Gersuk, H. A. DeBerg, E. Whalen, C. Ni, M. Farrington, D. Jeong, D. Robinson, P. S. Linsley, B. P. Vickery, W. W. Kwok, Sci. Transl. Med. 2017, 9, eaam9171; c) G. P. Fadini, D. Losordo, S. Dimmeler, Circ. Res. 2012, 110, 624; d) B. Zou, S. Sun, X. Qi, P. Ji, Mol. Med. Rep 2012, 5, 1116.

[5] a) X. Yang, R. Yao, H. Wang, Biomed. Res. Int. 2018; 9192104; b) S. Khorrami, Z. Hosseini, S. J. Mowla, R. Malekzadeh , Iran J Cancer Prev. 2015, 8, e3446. 
[6] H. M. Shapiro, Practical flow cytometry, Wiley-Liss, Hoboken, N.J., 2003.

[7] Flow cytometry: a practical approach (Ed.: M. G. Ormerod), Oxford University Press, New York, 2000.

[8] Live cell imaging: a laboratory manual (Eds.: R. D. Goldman, J. R. Swedlow, D. L. Spector), Cold Spring Harbor Laboratory Press, Cold Spring Harbor, N.Y., 2010.

[9] a) C. A. Schumann, A. Dörrenhaus, J. Franzke, P. Lampen, Anal. Bioanal. Chem. 2008, 392, 1159; b) X. Li, V. Ling, and P. C. H. Li, Anal. Chem. 2008, 80, 4095; c) E. Bedner, M. R. Melamed, Z. Darzynkiewicz, Cytometry 1998, 33:1.

[10] a) L. R. James, S. Andrews, S. Walker, P. R. S. de Sousa, A. Ray, N. A. Russell, T. C. Bellamy, PLoS ONE 2011, 6: e26889; b) T. P. Patela, K. Mana, B. L. Firestein, D. F. Meaneya, J. Neurosci. Meth. 2015, 243, 26; c) A. S. Neal, A. M. Rountree, J. R. Radtke, J. Yin, M. W. Schwartz, C. S. Hampe, J. D. Posner, V. Cirulli, I. R. Sweet, Scientific Reports 2016, 6, 39319.

[11] a) A. Zotter, F. Bäuerle, D. Dey, V. Kiss, G. Schreiber, J. Biol. Chem. 2017, 292, 15838; b) E. Durandau, D. Aymoz, S. Pelet, BMC Biol. 2015; 13, 55

[12] ABC transporters and multidrug resistance (Eds.: A. Boumendjel, J. Boutonnat, J. Robert): John Wiley \& Sons, Hoboken, N.J., 2009.

[13] Multi-drug resistance in cancer (Ed.: J. Zhou), Humana Press, New York, N.Y., 2010.

[14] I.V. Lebedeva, P. Pande, W. F. Patton, PLoS One 2011, 6, e22429.

[15] a) V. Richard, M. G. Nair, K. T. R. Santhosh, M. R. Pillai, Biomed. Res. Int 2013, 2013, 517237; b) B. Yu, D. Gu, X. Zhang, J. Li, B. Liu, J. Xie, Oncotarget 2017, 8, 27412.
[16] a) A. Golebiewska, N. H. C. Brons , R. Bjerkvig, S. P. Niclou, Cell Stem Cell 2011, 8, 136; b) S.-L. Mo, J. Li, Y. S. Loh, R. D. Brown, A. L. Smith, Y. Chen, D. Joshua, B. D. Roufogalis, G. Q. Li, K. Fan, M. C. H. Ng, D. Sze, Bone Marrow Res. 2011, 2011, Article ID 524845.

[17] F. J. Sharom, Biochem. Cell Biol. 2006, 84, 979.

[18] S. Michelson, D. Slate, Bull. Math. Biol. 1992, 54, 1023.

[19] a) C. Saengkhae, C. Loetchutinat, A. Garnier-Suillerot, Biochem. Pharmacol. 2003, 65, 969; b) I. E. Crompton, S. G. Waley, Biochem. J. 1986, 239, 221

[20] V. Koshkin, S. N. Krylov, PLoS One 2012, 7, e41368.

[21] G. Xu, Y. Zhong, S. Munir, B. B. Yang, B. K. Tsang, C. Peng, J. Clin Endocrinol. Metab. 2004, 89, 5523.

[22] L. Lacerda, L.s Pusztai, W. A. Woodward, Drug Resist. Updates 2010, 13, 99.

[23] B. Rychlik, A. Balcerczyk, A. Klimczak, G. Bartosz, J. Membr. Biol. 2003, 193 ,

[24] L. Payen, L. Delugin, A. Courtois, Y. Trinquart ,A. Guillouzo, O. Fardel O. Br J. Pharmacol. 2001, 132, 778

[25] D. N. Karunaratne, K. L. Audus, J. Natl. Sci. Found. Sri Lanka 2007, 35, 19.

[26] D. J. Holder, M. J. Marino, Curr. Protoc. Pharmacol. 2017, 76, A.3G.1.

[27] a) A. Nakano, D. Tsuji, H. Miki, Q. Cui, S. M. El Sayed, A. Ikegame, A. Oda, H. Amou, S. Nakamura, T. Harada, S. Fujii, K. Kagawa, K. Takeuchi, A. Sakai, S. Ozaki, K. Okano, T. Nakamura, K. Itoh, T. Matsumoto, M. Abe. PLoS One 2011, 6, e27222; b) Y. Teng, Y.Hsieh, C.Hung, H. Lin, J. Agric. Food Chem. 2015, 63, 847.

[28] A. R. Henderson, Clin. Chim. Acta. 2006, 366, 112.

[29] Y. A. Luqmani, Med. Princ. Pract. 2005, 14:35.

[30] H. Hochberg, Y. Brody, Y. Shav-Tal, Methods 2017, 120, 58 